\title{
Capsaicin induced cough in cryptogenic fibrosing alveolitis
}

\author{
M J Doherty, R Mister, M G Pearson, P M A Calverley
}

\begin{abstract}
Background-Cough is a common and troublesome symptom in cryptogenic fibrosing alveolitis (CFA) but the mechanisms responsible are not known. The cough threshold to inhaled capsaicin is increased in asthma and chronic obstructive pulmonary disease (COPD) where lung volumes are increased, but the relationship between cough response and symptom intensity has not been studied in CFA where lung volumes are reduced. Methods-Capsaicin challenge tests were performed on 15 subjects with proven CFA and 96 healthy controls. Symptoms, as assessed by daily diary card cough score and by visual analogue scale (VAS), were related to the capsaicin sensitivity (C5) and compared with lung volumes. Volume restriction was produced in a group of 12 normal healthy subjects by a plastic shell tightly strapped to the chest wall. Capsaicin challenge tests were performed in these subjects, both strapped and unstrapped, to determine whether volume restriction altered the cough reflex.
\end{abstract}

Results-The median C5 response in normal subjects was more than $500 \mu M$ compared with $15.6 \mu M$ in those with CFA $(p<0.001)$. The C5 response of the CFA patients was not related to symptoms of cough (whether measured by diary card or by VAS), nor was it related to percentage predicted total lung capacity (TLC) or forced vital capacity (FVC). Volume restriction of normal subjects with chest strapping successfully restricted lung volumes to levels similar to that of the CFA patients but did not change the sensitivity to capsaicin.

Conclusions-The cough reflex measured using capsaicin is markedly increased in patients with CFA. This increase is not the result of alterations in the deposition of inhaled particles of capsaicin brought about by volume restriction. It could be related to reduced lung compliance leading to sensitisation of rapidly adapting receptors, other mechanical changes, or to destruction of pulmonary $C$ fibres secondary to interstitial inflammation. The capsaicin test may be a useful method of objectively monitoring cough propensity in CFA.

(Thorax 2000;55:1028-1032)

Keywords: cryptogenic fibrosing alveolitis; cough threshold; capsaicin; volume restriction
Cryptogenic fibrosing alveolitis (CFA) is characterised by a mixture of inflammation and fibrosis of the pulmonary interstitium and air spaces $^{1}$ and has a prevalence of up to six per 100000 population. ${ }^{2}$ Patients with CFA usually have reduced lung volumes, a reduced transfer factor, and relatively preserved forced expiratory volume in one second $\left(\mathrm{FEV}_{1}\right)$, but this is not always present. ${ }^{3}$ Although breathlessness is the best recognised symptom, cough is reported by $86 \%$ of patients with CFA. ${ }^{4}$

Capsaicin (trans-8-methyl-N-vanillyl-6noneamide) is the pungent extract of red pepper which, when inhaled, induces cough reproducibly without tachyphylaxis. ${ }^{5}$ Inhalation of increasing doses of capsaicin is a sensitive way of assessing the cough reflex in a variety of disease states. ${ }^{6}$

Although cough is often reported by patients with CFA, the underlying mechanisms are not clear. Sputum production is infrequent and airway inflammation is not a prominent feature, unlike asthma and chronic obstructive pulmonary disease (COPD) where capsaicin sensitivity is increased. ${ }^{67}$ Cough receptors are thought to be present in proximal airways whereas the damage in CFA affects small airways and alveoli. At present the available data are conflicting and limited. In one study of 12 patients (four with CFA, eight with sarcoidosis) no abnormality in the cough response was found, ${ }^{6}$ although little information about the physiological and radiographic severity was included. In contrast, a larger study of patients with progressive systemic sclerosis (PSS) reported an increased cough response to capsaicin, and noted selfreported cough only in those patients with evidence of lung fibrosis. ${ }^{8}$

We hypothesised that capsaicin responsiveness might be increased in CFA for similar reasons to the patients with PSS, and that there should be a relationship between this increase and the severity of the restrictive disorder. Such changes might be a consequence of increased central airways deposition or loss of pulmonary stretch receptor inhibition of the cough reflex. To test this possibility we studied capsaicin responsiveness in a group of healthy volunteers before and during chest wall restriction sufficient to mimic the lung volumes experienced by patients with fibrosing alveolitis.

\section{Methods}

COMPARISON OF PATIENTS WITH CFA AND

HEALTHY CONTROLS

We studied 15 patients with CFA currently attending our outpatient clinics and 96 healthy individuals recruited from hospital staff and 
Table 1 Comparison of mean (SD) demographic factors in normal subjects, in the 14 oldest normal subjects, and in patients with cryptogenic fibrosing alveolitis (CFA)

\begin{tabular}{llll}
\hline & $\begin{array}{l}\text { Normal subjects } \\
(n=96)\end{array}$ & $\begin{array}{l}\text { Older normal subjects } \\
(n=14)\end{array}$ & $\begin{array}{l}\text { CFA } \\
(n=15)\end{array}$ \\
\hline Age (years) & $38(12)$ & $57(4)$ & $65(6)$ \\
$\%$ Male & 34 & 29 & 73 \\
$\%$ predicted FEV & $107(14)$ & $99(13)$ & $82(11)$ \\
$\%$ predicted FVC & $100(13)$ & $90(9)$ & $75(14)$ \\
TLCO & - & - & $57(20)$ \\
VAS cough & - & - & $3.0(1.9)$ \\
\hline
\end{tabular}

$\overline{\mathrm{FEV}_{1}}=$ forced expiratory volume in one second; FVC = forced vital capacity; TLCO = carbon monoxide transfer factor; VAS = visual analogue score.

relatives accompanying the patients. All the patients with CFA had been diagnosed on the basis of a clinical history, the presence of fine inspiratory basal crackles, and an open lung biopsy and/or a high resolution CT scan confirming the presence of pulmonary fibrosis without pleural disease. No patient had a history of asbestos exposure or clinical evidence of an underlying connective tissue disease or any other cause of pulmonary fibrosis. The normal subjects had neither symptoms nor a past history of any atopic disorder and all had spirometric values of $>88 \%$ predicted. ${ }^{9}$

No participant was under 18 years of age, had had a respiratory tract infection within the previous four weeks, was taking ACE inhibitors, or had current or previous symptoms suggestive of gastro-oesophageal reflux. All gave their informed consent and the study protocol was approved by the local research ethics committee.

\section{PROCEDURES}

Symptom scores

Symptomatic cough was measured in two ways in subjects with CFA: (1) Diary card: this was completed at home over the two weeks before the capsaicin test. The subjects recorded cough severity on a scale from $1=$ no cough to $5=$ distressing cough most of the day. This score was used to calculate the average daily diary cough score for each subject. The diary card data were accepted if it had been completed for at least 10 of the 14 days. (2) Visual analogue scale (VAS): each subject recorded the severity of their cough at their first attendance before any other testing using a $10 \mathrm{~cm}$ VAS ranging from no cough to worst cough imaginable.

\section{Capsaicin challenge test}

Each subject inhaled a single breath of solution via an Acorn nebuliser controlled by a custom made breath activated dosimeter. Saline was given first followed by doubling concentrations of capsaicin between 1.95 and $500 \mu \mathrm{M}$. The concentrations were given in ascending order and the subjects were not aware of the concentration they were inhaling. Each concentration was given at 30 second intervals and the number of coughs induced in this time was counted. The concentration that induced five or more coughs (C5) was recorded. If a subject did not cough five times on any of the concentrations this was also recorded.

The concentration at which the subject coughed five times was repeated and, if it was reproducible, the test was terminated. If it was not reproducible the test was continued until a reproducible $\mathrm{C} 5$ was found. The dosimeter had an in-built 0.25 second time delay and an inhalation time of two seconds, and during this time it delivered $0.009 \mathrm{ml}$ of nebulised solution. The mass median diameter (MMD) of the particles produced was $5.2 \mu \mathrm{m}$.

\section{Pulmonary function}

Spirometric parameters $\left(\mathrm{FEV}_{1}\right.$ and forced vital capacity (FVC)) were recorded in participants before capsaicin testing using a bellows spirometer and the best values from three technically acceptable attempts were used.

Static lung volumes including functional residual capacity (FRC), residual volume (RV), and total lung capacity (TLC) were measured using the helium dilution technique. Gas transfer factor (TLCO) was measured using a single breath method.

EFFECT OF CHEST WALL RESTRICTION IN HEALTHY SUBJECTS

Nine healthy adult volunteers (four men) were studied while free breathing and with their lung volumes restricted by wearing a tight fitting plastic shell around the chest wall and abdomen. The strapping was adjusted to reduce the FVC values to a level similar to that in the patients. Lung volume measurement and capsaicin testing were performed as described above on two occasions, either with or without strapping, the order being randomly assigned.

DATA ANALYSIS

Data are expressed as mean (SD). Cough symptom data were analysed nonparametrically throughout. C5 data are expressed as concentration steps and, when correlated with other data, the Spearman rank test was used as the distribution of the response in the patients with CFA was not normal. The Mann-Whitney U test was used to compare changes in lung volume and changes in C5 in healthy subjects during the strapping experiments. If five or more coughs were not achieved during the capsaicin challenge the C5 was denoted as the maximum concentration of capsaicin for the purposes of analysis. Given the differences in distribution of the ages of our normal population and the patients with CFA, a separate analysis of the older normal subjects is also presented for comparison.

\section{Results}

Demographic and spirometric data for the study populations are presented in table 1 . Patients with CFA were significantly older and more likely to be male than the healthy control group. To aid comparisons data are also presented on the 14 oldest members of the control population. Patients with CFA had a restrictive pattern of lung volumes with a significant reduction in TLCO in both comparisons. More CFA patients than controls were current or ex-smokers ( $73 \%$ vs $27 \%$ ).

Most patients with CFA reported symptoms of cough (mean (SD) VAS $3.0(1.9) \mathrm{cm}$ and average daily cough score $2.6(0.9)$ ). However, the severity of cough measured by these 


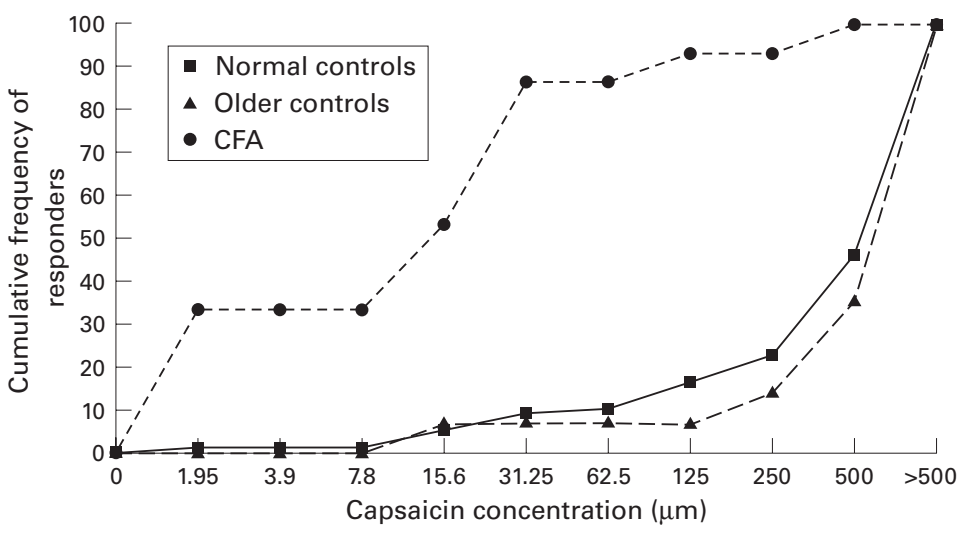

Figure 1 Cumulative frequency of the proportion with a positive capsaicin sensitivity (C5) response at each concentration of capsaicin for normal healthy controls, older healthy controls, and patients with cryptogenic fibrosing alveolitis (CFA).

Table 2 Mean (SD) lung volumes and C5 responses of normal subjects, both strapped and unstrapped, compared with patients with CFA

\begin{tabular}{llll}
\hline & Unstrapped $(n=9)$ & Strapped $(n=9)$ & CFA $(n=15)$ \\
\hline FVC \% predicted & $111(14)$ & $63(14)$ & $75(14)$ \\
FEV $_{1} \%$ predicted & $123(15)$ & $71(15)$ & $82(11)$ \\
RV \% predicted & $100(24)$ & $75(30)$ & $75(30)$ \\
TLC \% predicted & $101(16)$ & $67(15)$ & $74(16)$ \\
FRC (1) & $3.2(1.0)$ & $2.2(0.8)$ & $2.5(0.9)$ \\
IC (1) & $3.5(0.8)$ & $2.0(0.6)$ & $1.9(0.4)$
\end{tabular}

$\overline{\mathrm{FVC}}=$ forced vital capacity; $\mathrm{FEV}_{1}=$ forced expiratory volume in one second; RV = residual volume; TLC = total lung capacity; FRC = functional residual capacity; IC = inspiratory capacity.

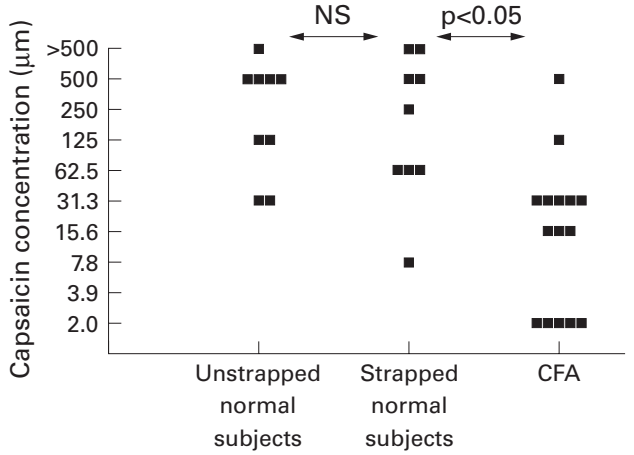
Figure 2 Comparison between the cough responses of
patients with cryptogenic fibrosing alveolitis (CFA) and normal subjects with and without chest strapping.

methods was in poor agreement and neither was related to the lung volumes.

The median C5 concentration was greater than $500 \mu \mathrm{M}$ (the highest concentration used) in normal subjects and $15.6 \mu \mathrm{M}$ in patients with CFA $(p<0.001)$. To exclude an age effect the $\mathrm{C} 5$ response was also calculated for the 14 oldest healthy subjects and was $500 \mu \mathrm{M}$ (fig 1). Neither current smoking habit nor sex affected these results in either the patient group or the healthy controls. There was no association between perceived symptoms of cough, however assessed, and the C5 concentration. Spirometric and lung volume indices and TLCO, whether expressed in absolute terms or as percentage predicted, were unrelated to the capsaicin sensitivity.

CHEST STRAPPING

The effects of chest wall restriction in healthy subjects are compared with data from CFA patients in table 2 . Restriction with the plastic shell produced significant reductions in all measurements of lung volume in the normal individuals and these were not significantly different from the resting data in the CFA subjects. Despite this, there was no change in the median $\mathrm{C} 5$ values of the normal subjects, which remained significantly different from those in the patients with CFA (fig 2).

\section{Discussion}

Cryptogenic fibrosing alveolitis is a disabling form of interstitial pulmonary fibrosis which appears to be becoming more frequent as the population ages. ${ }^{10}$ Although breathlessness is the dominant clinical symptom, chronic cough is frequently present as seen from our symptomatic assessment. Our data show a markedly enhanced cough responsiveness to capsaicin in patients with CFA which we hypothesise as being likely to reflect the presence of pulmonary fibrosis, although the precise mechanisms involved remain to be determined.

In patients with obstructive airways disease cough is well recognised and an increased C5 cough response to citric acid ${ }^{11}$ and capsaicin ${ }^{7}$ has been reported. The median cough threshold to capsaicin was 2-3 doubling dilutions less than normal in patients with $\mathrm{COPD}^{7}$ but was six doubling dilutions less in our patients with CFA. This marked increase is difficult to explain. One possibility could be a change in the distribution of the capsaicin aerosol. This might be a factor in the obstructed patients ${ }^{12}$ but is not likely to occur in restrictive disorders like CFA. ${ }^{13}$ The particles we used had an MMD of $5.2 \mu \mathrm{m}$ and should be deposited throughout the airways with a limited proportion reaching the alveoli. Previously we have conducted a radiolabelled deposition study using the same dosimeter in patients before and after chest strapping to a similar degree to that reported here, and we found no difference in the pattern of aerosol deposition (D Spence and D Pavia, personal communication), nor did we see any relationship between FVC, TLC, or the C5 response in our patients as might have been expected if altered structure leading to altered deposition were to explain the changes. Thus, while differences in the pattern of inhaled capsaicin deposition between normal subjects and those with disease might explain the increased sensitivity to capsaicin in obstructive airway disease, it seems unlikely to be an explanation in CFA.

To date, there is only limited information about cough symptoms and the response to tussive agents in interstitial lung disease. The VAS cough score in our patients was very similar to that in the PSS patients with fibrosis studied by Lalloo et al, ${ }^{8}$ but significantly less than that reported in patients with chronic unexplained cough. The capsaicin challenge we used was similar to that reported previously ${ }^{6}$ and we adapted the method of previous studies in assigning an arbitrary value to the peak concentration administered. We did not report the concentration provoking two coughs (C2) as we found it less reproducible and it produced a less clear distinction between health and disease. The mean $\mathrm{C} 5$ value in the normal sub- 
jects was higher than that reported by Lalloo et $a l^{8}$ but the distinction between normal subjects and patients with CFA is clearly demonstrated. This difference in control values is likely to reflect differences in dosimeter output (MMD of particles $4.0 \mu \mathrm{m}$ in the study by Lalloo et $\left.a l^{8}\right)$, a variable known to increase capsaicin responsiveness in normal subjects. ${ }^{14}$ As expected, the patients were older than our normal subjects and most were men, making exact comparisons with this group population difficult. However, we found no relationship in the healthy individuals between C5 and age, sex, or smoking habits, nor did we see any differences between the older subjects and the population as a whole. This has been reported previously when using a similar dosimeter methodology, ${ }^{6}$ but has not been found in all studies. ${ }^{15}{ }^{16} \mathrm{We}$ chose our patients irrespective of their complaint of cough and as consecutive individuals with CFA attending our clinic who met the entry criteria to the study. Thus, we believe the differences in C5 threshold seen in the patient group is a feature of the illness and not of patient selection.

The lack of relationship in the perception of cough measured on the visual analogue scale and the mean diary card score was disappointing. This may reflect the relatively small number of patients studied, but it is similar to our experience in a larger population of patients with COPD. ${ }^{7}$ This emphasises the difficulty in quantifying a chronic symptom like cough, where adaptation to its presence occurs over time and where there may be a confounding effect of the baseline intensity of the symptom on the patient's perception of severity, as is the case with breathlessness. ${ }^{17}$ The lack of any association with C5 may reflect these adaptive processes but it is clear that cough reflex sensitivity is only one factor responsible for symptomatic cough. Coughing may be influenced by recent respiratory tract infections and by changes in temperature which interact with the cough threshold to cause symptomatic coughing spells.

We noted weak associations between the VAS cough score and the $\mathrm{FEV}_{1} / \mathrm{FVC}$ ratio $(r=0.33)$ together with a trend for a similar association with C5 (unlike the healthy controls) which raised the possibility that reduced lung volumes per se might explain the altered cough responsiveness. Methacholine reactivity is increased by chest strapping and by the associated reduction in pulmonary elastic recoil with loss of airway parenchymal interaction. ${ }^{18}$ This led to our study of the effects of lung volume restriction by strapping the chests of normal subjects. Although we successfully simulated the reduced lung volumes experienced by our patients, there was no change in the C5 response of the healthy volunteers. This protocol reduced effective chest wall compliance but without changing pulmonary compliance, and excluded an immediate reflex increase in cough responsiveness. However, it was not sustained long enough for secondary pulmonary changes such as microatelectasis to develop. Animal studies suggest that reduced lung compliance increases the firing rate of rapidly adapting receptors which some, ${ }^{19}$ but not all, ${ }^{20}$ believe are important in capsaicin induced cough.

Two further alternatives are worth considering. Although most pathological studies focus on the intense inflammation and fibrosis around the alveolar structures in patients with CFA, one report has commented on the presence of inflammation in the airways. ${ }^{4}$ However, the normal C5 data from patients with sarcoidosis, where airway inflammation is common, argue against inflammation alone being important. More plausible is a role for pulmonary fibrosis in causing destruction of the pulmonary C fibres. ${ }^{8}$ Stimulation of pulmonary rather than bronchial C fibres inhibits the cough response in animal studies. ${ }^{21}$ Moreover, our patients were selected for the presence of histological and/or CT evidence of fibrosis. The distribution of these changes in CFA is identical to that in PSS $^{22}$ where fibrosis was also associated with a reduced C5 value, in contrast to sarcoidosis where preserved capsaicin responses were found. ${ }^{6}$ It will be difficult to distinguish these effects in humans from those caused by rapidly adapting receptor damage, but it might be possible using careful CT staging of the pattern of parenchymal damage. ${ }^{23}$ However, technical differences between the diagnostic scans precluded this assessment in our patients.

Our study provides further evidence that cough is a common symptom in CFA and suggest that reflex regulation of cough is markedly altered in this disease. Whether these findings are due to mechanical disruption or to specific disease processes is unknown. Similarly, whether they have prognostic importance remains to be determined. The high frequency with which an abnormal capsaicin sensitivity is found in these patients makes this non-invasive test a potentially useful way of monitoring the effects of treatment on this distressing symptom.

This work was supported in part by the University Hospital Aintree Research and Development programme. MJD was in Aintree Research and Development programme. MJD was in
receipt of a fellowship provided by the Fazakerley Fund for Respiratory Research.

piratory Research.
Conflicts of interest: none.

$1 \mathrm{Du}$ Bois RM. Cryptogenic fibrosing alveolitis and cryptogenic organising pneumonia. In: Brewis RAL, Corrin B, Gibson GJ, Geddes DM, eds. Textbook of respiratory medicine. 2nd ed. London: Bailliere Tindall, 1995.

2 Scott J, Johnston IDA, Britton J. What causes cryptogenic fibrosing alveolitis? A case control study of environmental exposure to dust. BMF 1990;301:1015-7.

3 Doherty MJ, Pearson MG, O'Grady EA, et al. Cryptogenic fibrosing alveolitis with preserved lung volumes. Thorax 1997;52:998-1002.

4 Crystal RG, Fulmer JD, Roberts WC, et al. Idiopathic pulmonary fibrosis: clinical, histological, radiographic, physiological, scintigraphic, cytological and biochemical aspects. ological, scintigraphic, cytological

5 Morice AH, Higgins KS, Yeo WW. Adaptation of cough Morice AH, Higgins KS, Yeo WW. Adaptation of cough
reflex with different types of stimulation. Eur Respir f 1992; reflex with

6 Choudry NB, Fuller RW. Sensitivity of the cough reflex in patients with chronic cough. Eur Respir F 1992;5:296-300. 7 Doherty MJ, Mister R, Pearson MG, et al. Capsaicin responsiveness and cough in asthma and chronic obstructive pulmonary disease. Thorax 2000;55:643-9.

8 Lalloo UG, Lim S, DuBois R, et al. Increased sensitivity of the cough reflex in progressive systemic sclerosis patients with interstitial lung disease. Eur Respir f 1998;11:702-5.

9 Quanjer PH, Tammeling GJ, Cotes JE, et al. Lung volumes and forced ventilatory flows. Report of Working Party on and forced ventilatory flows. Report of Working Party on Standardization of Lung Function Tests, European Compean Respiratory Society. Eur Respir $\mathcal{F}$ (Suppl) 1993;16:540 .

10 Hubbard R, Johnston IDA, Coultas DB, et al. Mortality rates from cryptogenic fibrosing alveolitis in seven countries. Thorax 1996;51:711-6. 
11 Wong $\mathrm{CH}$, Morice $\mathrm{AH}$. Cough threshold in patients with chronic obstructive pulmonary disease. Thorax 1999;54 $62-4$.

12 Love RG, Muir DCF. Aerosol deposition and airway obstruction. Am Rev Respir Dis 1976;114:891-7.

13 Anderson PJ, Wilson DJ, Hiller CF. Respiratory tract deposition of ultrafine particles in subjects with obstructive or restrictive lung disease. Chest 1990;97:1115-20.

14 Dicpringaitis PV, Rauf $\mathrm{K}$. The influence of gender on cough reflex sensitivity. Chest 1998;113:1319-21

15 Fujimura M, Kasahara K, Kamio Y, et al. Female gender as a determinant of cough threshold to inhaled capsaicin. Eur Respir ₹ 1996;9:1624-6.

16 Hanson L, Wolliner P, Dahlback M, et al. Regional sensitivity of human airways to capsaicin-induced cough. Am Rev Respir Dis 1992;145:1191-5.

17 Lansing RW, Banzett RB. Psychophysical methods in the study of respiratory sensation. In: Adams L, Guz A, eds.
Respiratory sensation. New York: Marcel Dekker, 1996 .
18 Ding DJ, Martin JG, Macklem PT. Effects of lung volumes on maximal methacholine induced bronchoconstriction in on maximal methacholine induced bronchoconstriction humans. $\mathcal{A}$ Appl Physiol 1987;62:1324-30.

19 Karlsson JA, Sant'Ambrogio G, Widdicombe J. Afferent neural pathways in cough and reflex bronchoconstriction. $\mathcal{F}$ Appl Physiol 1988;65:1007-23.

20 Collier JG, Fuller RW. Capsaicin inhalation in man and the effects of sodium cromoglycate. Br F Pharmacol 1984;81: 113-7.

21 Tatar M, Webber SE, Widdicombe JG. Lung C-fibre receptor activation and defensive reflexes in anaesthetized cats. $\mathcal{F}$ Physiol (Lond) 1988;402:411-20.

22 Wallaert B, Perez T, Tonnel AB. The lung in rheumatological diseases. In: Wallers EH, Du Bois RM, eds. Immunology and management of interstitial lung diseases. London: Chapman and Hall, 1995.

23 Wells A, Rubens MB, Du Bois RM, et al. Serial CT in fibrosing alveolitis: prognostic significance of the initial pattern. SfR 1993;161:1159-65. 